\title{
A NOVEL STRATEGY FOR RADAR IMAGING BASED ON COMPRESSIVE SENSING
}

\author{
Mariví Tello, Paco López-Dekker, Jordi J. Mallorquí \\ Remote Sensing Laboratory (RSLAB), Universitat Politecnica de Catalunya (UPC)
}

\begin{abstract}
This paper aims at introducing the recent theory of compressive sensing to radar imaging systems in order to retrieve the imaged scene with better resolution and a reduced amount of collected samples. As a result of the application of the alternative imaging technique proposed, the use of matched filtering is avoided and the effect of its sidelobes in the images is drastically diminished. Furthermore, the amount of data to be stacked in the sensor and then downlinked to the ground station is meaningfully lower. This permits a more efficient management of resources.
\end{abstract}

Index Terms - compressive sensing, radar imaging, matched filter, matching pursuit, sparseness, compressibility.

\section{INTRODUCTION}

A radar system aims at acquiring a representation as accurate as possible of the backscattering properties of the illuminated scene. This is achieved by transmitting a pulse and then analyzing the echo reflected back to the receiver, following the basic configuration depicted in Fig. 1. This configuration is fixed, but there is still freedom in the design of the transmitted pulse $s_{T}(t)$ and of the strategy used in reception for the analysis of the collected echoes $s_{R}(t)$. Until now, the most widely used strategy, is the one that sends a signal and then compresses it by means of matched filtering on the receiver [1]. This paper proposes an alternative of design based on the concepts introduced by the theory of Compressive Sensing (CS), recently developed in the fields of information theory and signal processing.

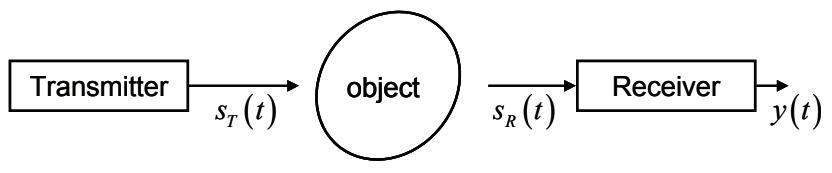

Figure 1. Basic configuration of a radar system.
Next section will briefly review and discuss the conventional radar imaging system. Then, the basis of the theory of CS will be presented in section III. Based on the observations raised in previous sections, Section IV will propose a novel strategy for radar imaging through analytic expressions and preliminary results will be shown.

\section{CONVENTIONAL RADAR ACQUISITION: DISCUSSION OF MAIN DRAWBACKS}

Essentially, a typical radar system operates by transmitting a wideband pulse which is compressed later on, by correlating the received echo with the corresponding matched filter [1,2]. Since short pulses lead to high resolution but pose a constraint for the transmitted power, a convenient solution is to use a long pulse modulation, e.g., with a linear frequency modulation (chirp function). With the notation introduced in Fig. 1.:

$$
s_{T}(t)=\exp \left\{j\left(\omega_{0} t-\beta t^{2}\right)\right\} \text { for }|t| \leq \tau_{p} / 2
$$

where $\omega_{0}$ is the carrier frequency and $\tau_{p}$ is the pulse duration. With matched filtering, the signal representing the backscattering properties of the illuminated scene $y(t)$ is obtained as the convolution of the received signal with its complex conjugated, time-reverted function:

$$
\begin{aligned}
& y(t)=\int_{-\tau_{,} / 2}^{\tau_{r} / 2} \exp \left(j \beta s^{2}\right) \exp \left(-j \beta[s+t]^{2}\right) \operatorname{rect}\left(\frac{s+t}{\tau_{p}}\right) d s \\
& \approx \operatorname{sinc}\left(\frac{\beta}{\pi} t\left[\tau_{p}-|t|\right]\right)
\end{aligned}
$$

Matched filtering results from the design criterion stated consisting on the maximization of the signal to noise ratio if assuming additive white noise. From this point of view, this is the optimum solution. The main drawback of matched filtering with a chirp signal is the existence of sidelobes eventually preventing the discrimination of targets close to each other. Additionally, such a design requires a high rate $\mathrm{A} / \mathrm{D}$ converter at reception. This sampling operation is expensive and produces a huge 
amount of data which greatly occupies storage and downlink bandwidth resources.

The option of design reviewed in this section is based on the classical theory of Shannon and on the Nyquist theorem for signal sampling. The different alternative proposed in section IV searches a better use of the available bandwidth through CS tools which constitute an alternative to Shannon theory.

\section{COMPRESSIVE SENSING: THEORETICAL BASIS}

CS is a signal processing theory developed recently [3]. This section is devoted to the statement of its principles.

Consider a finite signal $x \in \mathbb{R}^{N}$ and a limited number of observations in the form of $K$ linear measurements:

$$
y_{k}=\left\langle x, \psi_{k}\right\rangle, k=1, \ldots, K
$$

where $\psi_{k}$ are known test signals. Equivalently, in matrix notation:

$$
y=\Phi x
$$

where $\psi_{k}$ are the rows of $\Phi$. In the case there are many more unknowns than observations $(K<<N)$, the system is highly undetermined and no direct solution is expected. Nevertheless, it has been shown that it is very likely to recover $x$ exactly provided that it is sparse and that matrix $\Phi$ obeys a Restricted Isometry Property (RIP), by means of convex programming:

$$
(P 1) \min _{\tilde{x} \in \mathbb{R}^{N}}\|\tilde{x}\|_{l_{1}} \text { subject to } y=\Phi \tilde{x}
$$

On the one hand, RIP is fulfilled if the columns of $\Phi$ are approximately orthogonal. On the other hand, a signal $x$ is sparse if there exists a domain $\psi$ in which the coefficient sequence is supported on a small set. It has been shown [4] that this reasoning can be extended to compressible and to complex signals, with independence of their dimension. Compressibility is a condition far less restrictive than sparsity and most of natural and manmade signals accomplish it.

\section{NOVEL STRATEGY FOR RADAR IMAGING}

This section is devoted to the presentation of the technique proposed in this paper for radar imaging. First, the theoretical principles of the recovery algorithm will be developed. Then, illustrative results on simulated data will be shown. Finally, an example of application on raw data from ERS sensor will be addressed.

\subsection{Design of the recovery algorithm}

Under the Born hypothesis, it can be assumed that the signal returned to the sensor can be modeled as the convolution of $s_{T}(t)$ with the reflectivity of the observed scene $r(t)$.

$$
s_{R}(t)=s_{T}(t) * r(t)=\int_{-\infty}^{\infty} s_{T}(t-\tau) r(\tau) d \tau
$$

In the discrete domain, different sampling intervals $k_{1}$ and $k_{2}$ for $t$ and $\tau$ respectively can be considered. Thus, $t=k_{1} n, \quad \tau=k_{2} m$ and the convolution in the discrete domain can be expressed as:

$$
s_{R}\left(k_{1} n\right)=\sum_{m} s_{T}\left(k_{1} n-k_{2} m\right) r\left(k_{2} m\right)
$$

If dealing with finite length signals (pulse duration $\tau_{p}$ is not infinite), the convolution can be expressed as a product of a matrix $S$ with a vector $r$ :

$$
s_{R}=S \cdot r
$$

$\left[\begin{array}{c}s_{R}\left(k_{1}\right) \\ s_{R}\left(2 k_{1}\right) \\ \vdots \\ s_{R}\left(k_{1}\right) \\ s_{R}\left((N+1) k_{1}\right) \\ \vdots \\ s_{R}\left((2 N-1) k_{1}\right) \\ s_{R}\left(2 N k_{1}\right)\end{array}\right]=\left[\begin{array}{cccc}s_{T}\left(k_{2}\right) & 0 & \cdots & 0 \\ s_{T}\left(2 k_{2}\right) & s_{T}\left(k_{2}\right) & \cdots & 0 \\ \vdots & \vdots & \ddots & \vdots \\ s_{T}\left(M k_{2}\right) s_{T}\left({ }_{(M-1) k_{2}}\right) \ldots & s_{T}\left(k_{2}\right) \\ 0 & s_{T}\left(M k_{2}\right) & \cdots & s_{T}\left(2 k_{2}\right) \\ \vdots & \vdots & \ddots & \vdots \\ 0 & 0 & \cdots s_{T}\left((M-1) k_{2}\right) \\ 0 & 0 & \cdots & s_{T}\left(M k_{2}\right)\end{array}\right]\left[\begin{array}{c}r\left(k_{2}\right) \\ r\left(2 k_{2}\right) \\ \vdots \\ r\left(M k_{2}\right) \\ r\left((M+1) k_{2}\right) \\ \vdots \\ r\left((2 M-1) k_{2}\right) \\ r\left(2 M k_{2}\right)\end{array}\right]$

With $M$ and $N$ expressed respectively as $N=\tau_{p} / k_{1}$ and $M=\tau_{p} / k_{2}$. In reception, the goal is to infer as closely as possible $r(n)$ through the samples $s_{R}(n)$. In the context of radar processing together with $\mathrm{CS}$, on the one hand, $S$ is a band matrix and, as a consequence, it satisfies RIP. On the other hand, it is reasonable to assume valid the hypothesis of compressibility of radar data [5]. Hence, conditions to apply CS are fulfilled. Nevertheless, the main interest of CS techniques is that of solving highly undetermined systems $y=\Phi x$, i.e. with matrix $\Phi$ having much more columns than rows. So, in order to bring $s_{R}=S \cdot r$ to a CS scheme, two options have been explored in the framework of this paper.

The first one consists on reducing the amount of samples collected in the receiver. By doing so, in the formulation of the system of linear equations, $s_{R}=S \cdot r$, just the rows of $S$ corresponding to the samples of $r$ collected are to be considered. This results on an undetermined $y=\Phi x$ system. As it has been seen in section III, in such a situation, $x$ can be retrieved exactly with a number of measurements of the order of $\alpha \log (N)$, being $\alpha$ the order of sparsity of $x$. Technologically, in the 
context of radar imaging, this implies recovering the imaged scene, avoiding the use of the matched filter, sampling in the receiver at a drastically lower rate than that accomplishing Nyquist (thus highly reducing the amount of data to be stacked in the sensor and downloaded to the ground station). The difficulty is then shifted from hardware to processing.

The second approach considered proposes taking into account the entire vector $s_{R}$, but assuming that $k_{2}<k_{1}$, when building matrix $\Phi$ (this is easily feasible since the receiver is aware of the transmitted waveform). An estimation of the imaged scene is then obtained with a higher number of samples, i.e., with a better resolution.

\subsection{Results on simulated data}

In order to check the validity of the alternative radar imaging system exposed in this paper, a first experiment has been carried out on a set of simulated 1D $s_{R}$ signals. The procedure of the simulation is the following. The round trip delay for a collection of targets is randomly generated using a uniform distribution corresponding to a range of valid distances. Then the scattering coefficient of each target is randomly chosen from a zero mean normal distribution. The received signal is generated as the sum of the time shifted replicas of the transmitted waveform, each multiplied by its respective scattering coefficient. To allow delays corresponding to a fraction of a sample, time shifting is performed in the frequency domain. Once the received signal has been synthesized, a random noise vector is added to simulate thermal noise. Then, just a fraction of samples of this simulated received signal has been kept (it is worth noting that these samples can be taken randomly) and the scene has been recovered considering a CS problem, i.e., through solving a convex linear problem as in (P1) with a matching pursuit algorithm. In order to evaluate the possibilities of this new radar imaging technique in relation to existing ones, the processing has also been carried out with matched filtering and results are compared. Results are shown in Fig. 2.

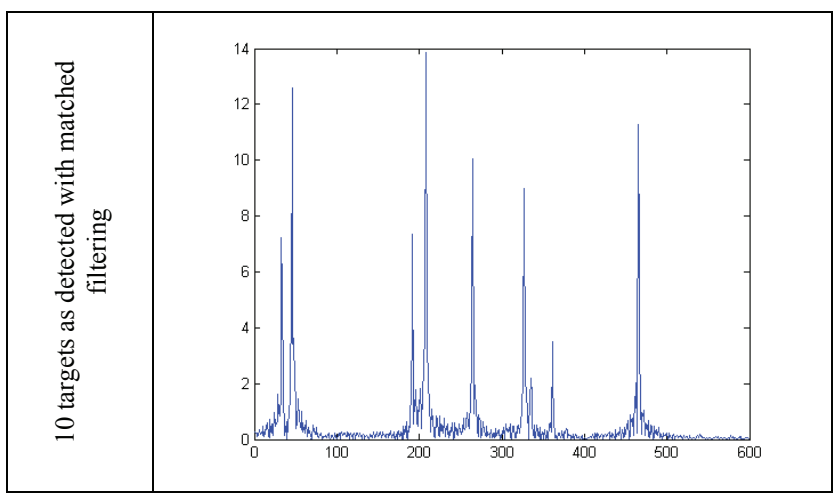

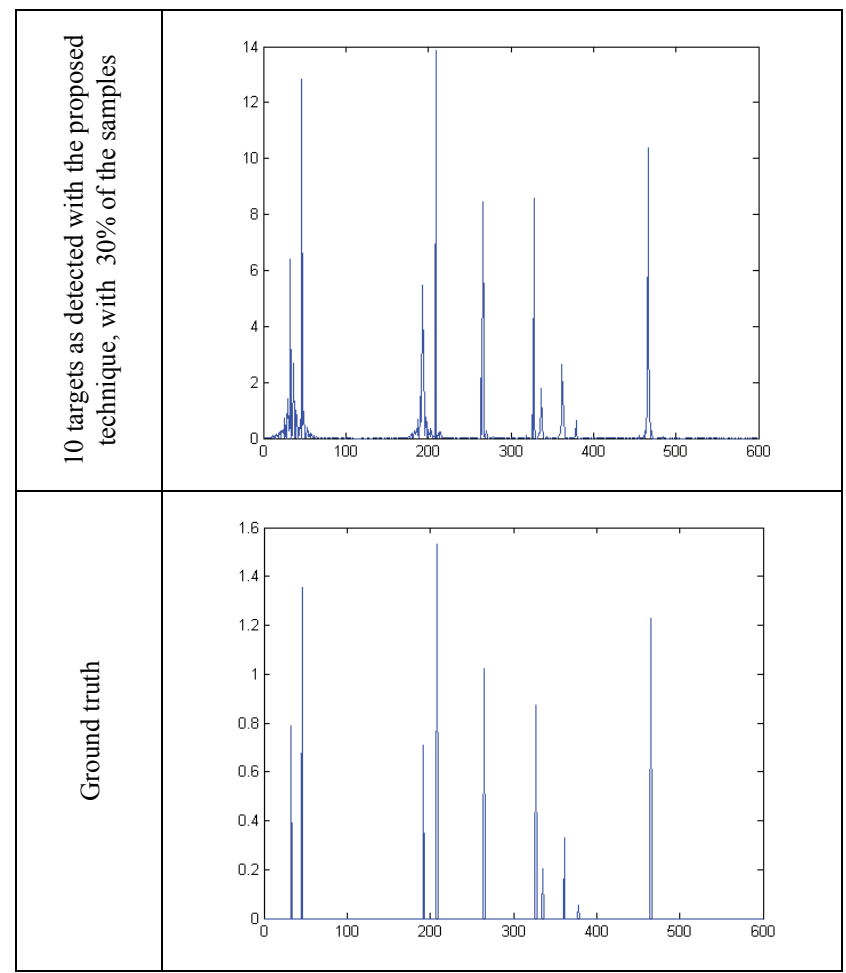

Figure 2. Recovery of a simulated scene with ten point targets, with no noise. (top) Recovery by means of matched filtering. (bottom) Recovery by means of the proposed technique, just taking $30 \%$ of the samples.

A noticeable reduction of the sidelobes due to matched filtering can be appreciated in the solution with the alternative radar imaging technique proposed. This permits detecting targets that are masked otherwise using traditional methods (see second target from the right in Fig. 2).

\subsection{Results on real data}

A second experiment has been completed on raw data acquired with the ERS sensor. On a first step, the procedure applied consists on processing the data in the range direction by means of conventional compression with matched filtering and then focusing in azimuth with the technique proposed based on CS. Just a $20 \%$ of the lines in azimuth have been considered for processing (see Figs. 34).

The objective of this experiment is to illustrate an example of application of the new method, regarding SAR focusing process. In fact, the scenario considered can be assimilated to a conventional wideswath acquisition mode in which just a fraction of samples is azimuth are taken into account to enlarge the swath, but it is interesting to notice that the resolution is not degraded when focusing in azimuth with the alternative strategy proposed. 

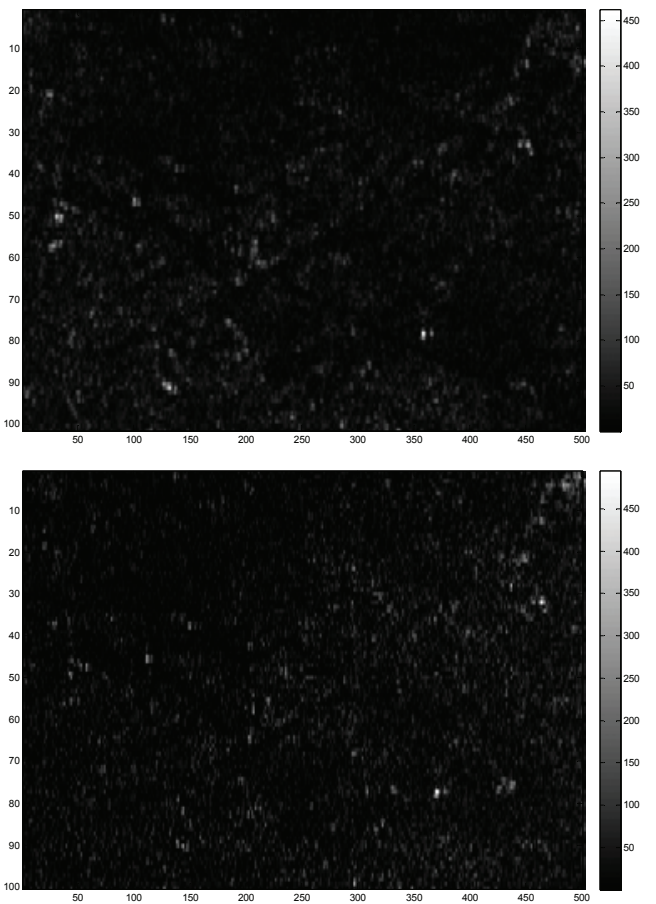

Figure 3. Focusing of raw ERS data, corresponding to a urban scene. (Top) Focusing by means of matched filtering in both range and azimuth (Bottom) Focusing by means of matched filtering in range and CS in azimuth.
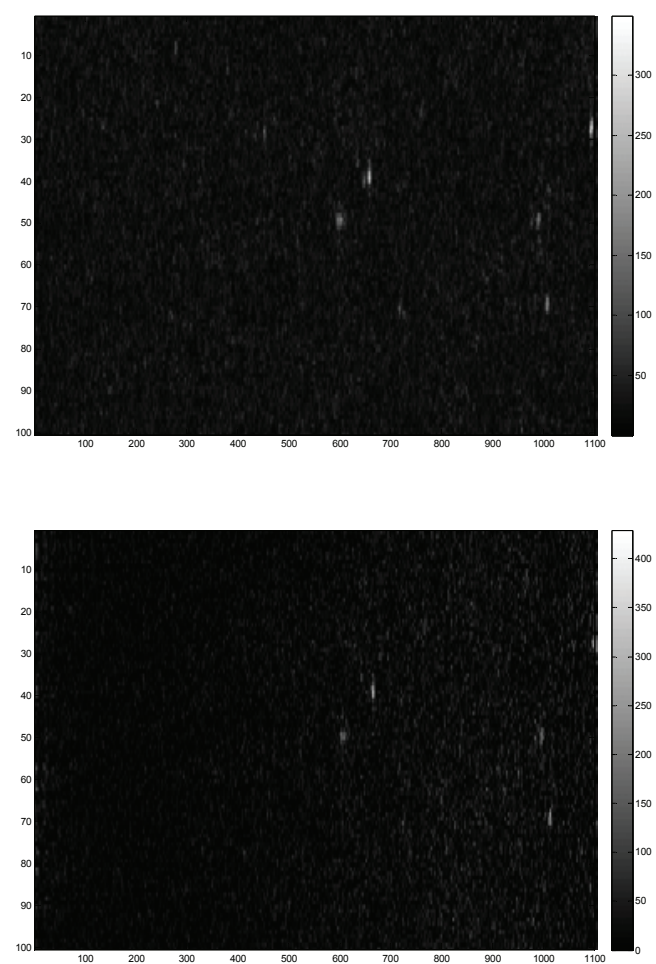

Figure 4. Focusing of raw ERS data, corresponding to an oceanic scene with targets corresponding to ships. (Top) Focusing by means of matched filtering in both range and azimuth (Bottom) Focusing by means of matched filtering in range and CS in azimuth.

\section{CONCLUSIONS}

A novel radar imaging approach has been exposed in this paper. It is based on CS theory which aims at solving highly undetermined systems through convex linear programming, taking advantage of the fact that most natural and manmade signals are compressible. Preliminary simulations on simple 1D scenes show a better performance of CS techniques respect to traditional matched filtering. Moreover, these results have been obtained by taking into account all the available samples at the input of the matched filter, but just a highly reduced amount when using CS. The implications of such a result on radar technology are meaningful. The sampling rate at the receiver (which is from a technological point of view a critical issue) can be dramatically reduced, such as the amount of data having to be stacked in the sensor and then transmitted to the ground station. Additionally, the technique proposed has been applied to real SAR data. An example of alternative focusing of ERS raw data in the azimuth direction has been tested.

This work was supported by the Spanish MCYT and FEDER funds under project TEC2005-06863-C02-01.

\section{REFERENCES}

[1] Levanon, N., Mozezon, E., Radar Signals, Wiley, 2004.

[2] Oliver, C., Quegan, S., Understanding Synthetic Aperture Radar Images, Artech House Publishers, 1998.

[3] Donoho, D., "Compressed Sensing", IEEE Trans. On Information Theory, 52(4), pp. 1289-1306, April 2006.

[4] Donoho, D., Tsaig, Y., "Extensions of compressed sensing", IEEE Trans. On Signal Processing, 86(3), pp. 533-548, March 2006.

[5] Valade, C., Nicolas, J.M., "Homomorphic Wavelet Transform and new subband statistics models for SAR image compression", IEEE Trans. On Signal Processing, 86(3), pp. 533-548, March 2006. 